\title{
Neutrino Mass Matrices in Models with Horizontal Symmetries
}

\author{
Asim K. Ray and Utpal Sarkar \\ Department of Physics, Visva-Bharati University, Santiniketan 731 235, \\ India
}

\begin{abstract}
We have studied the most general neutrino mass matrices in models with $\mathrm{SU}(2)$ and $\mathrm{SU}(3)$ horizontal symmetries. Without going into the details of the models it is possible to write down the effective operators, which predict the structure of the Majorana neutrino mass matrices. Unlike other extensions of the standard model, the structure is now independent of the effective Yukawa couplings and depends entirely on the Higgs which gives mass to the other fermions. In the case of $\mathrm{SU}(3)$ symmetries the lowest dimensional operators are forbidden requiring a low mass scale for lepton number violation.
\end{abstract}


The structure of fermion masses and mixing is not predicted in the standard model because all generations are treated similarly. All the fermion masses and mixing are free parameters, which are supposed to be determined by experiments. To eliminate this uncertainty and to understand the generation structure several approaches has been considered [1]. One such approach assumes that the fermions of different generations are related by some gauge symmetry group, called the horizontal symmetry [2]. The vacuum expectation values (vev) of the Higgs would then give us the structure of the fermion masses and mixing.

So far most of the searches for physics beyond the standard model has met with negative results except for the neutrino sector. The neutrino mass is predicted to be zero in the standard model, but a solution to the atmospheric neutrino anomaly [3] has evidenced a nonvanishing mass for the neutrinos. This result has been supported by the solar neutrino results [四]. Even the Laboratory experiments started supporting this result predicting a neutrino mass and mixing required to explain the observed neutrino oscillations [5, 6]. The developments in the neutrino physics over the past few years have already established some possible structures for the neutrino mass matrix.

Since the horizontal symmetric models are some of the possible extensions of the standard model, it is most natural to study the horizontal symmetries in the light of the recent results from neutrino physics. In this article we point out that it is possible to make some general comments about the neutrino masses and mixing in some of the models of gauged horizontal symmetries. We shall discuss the nonabelian $S U(2)_{H}, S U(3)_{H}^{V}$ and $S U(3)_{H}^{V L}$ horizontal gauge symmetries, although our results could be generalized to other symmetries as well.

We start with a brief introduction to the horizontal symmetries, we are going to discuss. We shall include the right handed neutrinos and their interactions while studying the neutrino masses. In the $S U(2)_{H}$ horizontal symmetry all the left and right handed fermions and antifermions transform 
as triplets. The fermion bilinears can thus be a singlet, tripplet or a 5-plet and hence the standard model Higgs doublet fields required to give fermion masses have to transform as a singlet $\phi^{a 1}[1,2,1,1]$ (trace), triplet $\phi_{k}^{a 2}[1,2,1,3]$ (antisymmetric tensor) or a 5 -plet $\phi_{i j}^{a 3}[1,2,1,5]$ (traceless symmetric tensor of rank 2) under $S U(2)_{H}$. So, we need one or more of the Higgs scalars $\phi^{a 1}$, $\phi_{i}^{a 2}$ or $\phi_{i j}^{a 3}$ to write down the Yukawa couplings for the fermion masses and mixing

$$
\begin{aligned}
\mathcal{L}_{Y}= & f_{1}^{u} \delta_{i j} \bar{q}_{i L} u_{j R} \phi^{a 1^{\dagger}}+\epsilon_{i j k} f_{3}^{u} \bar{q}_{i L} u_{j R} \phi_{k}^{a 2^{\dagger}}+f_{5}^{u} \bar{q}_{i L} u_{j R} \phi_{i j}^{a 3 \dagger} \\
& +f_{1}^{d} \delta_{i j} \bar{q}_{i L} d_{j R} \phi^{a 1}+\epsilon_{i j k} f_{3}^{d} \bar{q}_{i L} d_{j R} \phi_{k}^{a 2}+f_{5}^{d} \bar{q}_{i L} d_{j R} \phi_{i j}^{a 3} \\
& +f_{1}^{e} \delta_{i j} \bar{\ell}_{i L} e_{j R} \phi^{a 1}+\epsilon_{i j k} f_{3}^{e} \bar{\ell}_{i L} e_{j R} \phi_{k}^{a 2}+f_{5}^{e} \bar{\ell}_{i L} e_{j R} \phi_{i j}^{a 3}+\text { h.c. }
\end{aligned}
$$

Here $i, j, k=1,2,3$ are $S U(2)_{H}$ indices. The vevs of the different components of a Higgs scalar now determine the structure of the fermion mass matrices. The $S U(2)_{H}$ symmetry now requires same Yukawa couplings for all generations. The singlet $\phi^{a 1} \delta_{i j}$ being the trace of the matrix in the generation space, a vev of the field $\phi^{a 1}$ can only give equal masses to all the fermion generations with no mixing. Thus we need at least two or more of the Higgs fields $\phi_{k}^{a 2}$ and $\phi_{i j}^{a 3}$. In addition to these standard model Higgs doublets there are some singlets fields which breaks the horizontal group. Their inclusion do not change our analysis.

There are two possible realizations of the $S U(3)$ horizontal symmetries. In the vector-like case the left handed fermions and antifermions transform as triplets, while the right handed fermions and antifermions transform as antitriplets of $S U(3)_{H}^{V L}$. In this case we need to introduce mirror fermions to make the theory anomaly free and hence consistent. For every left handed field there is a right handed mirror field which has the same transformation under all gauge symmetries. In some theories such mirror fermions come out naturally and has interesting consequences, but otherwise this doubles the number of fermions. The mirror fermions are decoupled by some discrete symmetry from interacting with usual fermions and hence escape all 
detections. We shall not discuss the mirror sector here.

To contribute to the fermion masses the standard model Higgs doublet now could be an antitriplet $\phi_{[i j]}^{b 1}[1,2,1, \overline{3}]$ (antisymmetric rank 2 tensor) or a sextet $\phi_{\{i j\}}^{b 2}[1,2,1,6]$ (symmetric rank 2 tensor) under $S U(3)_{H}^{V L}$. To give masses to both the up and down quark sector we need another triplet $\tilde{\phi}_{[i j]}^{b 1}[1,2,1,3]$ and antisextet $\tilde{\phi}_{[i j]}^{b 2}[1,2,1, \overline{6}]$ of $S U(3)_{H}^{V L}$, which carry same hypercharge as $\phi_{\{i j\}}^{b 1}$ and $\phi_{[i j]}^{b 2}$ respectively. The Yukawa couplings are now given by

$$
\begin{aligned}
\mathcal{L}_{Y}= & h_{3}^{u} \bar{q}_{i L} u_{j R} \tilde{\phi}_{[i j]}^{b 1^{\dagger}}+h_{3}^{d} \bar{q}_{i L} d_{j R} \phi_{[i j]}^{b 1}+h_{3}^{e} \bar{\ell}_{i L} e_{j R} \phi_{[i j]}^{b 1} \\
& +h_{6}^{u} \bar{q}_{i L} u_{j R} \tilde{\phi}_{\{i j\}}^{b 2^{\dagger}}+h_{6}^{d} \bar{q}_{i L} d_{j R} \phi_{\{i j\}}^{b 2}+h_{6}^{e} \bar{\ell}_{i L} e_{j R} \phi_{\{i j\}}^{b 2}+\text { h.c. }
\end{aligned}
$$

Since $\phi_{[i j]}^{b 1}\left[\tilde{\phi}_{[i j]}^{b 1}\right]$ and $\phi_{\{i j\}}^{b 2}\left[\tilde{\phi}_{\{i j\}}^{b 2}\right]$ are most general anti-symmetric and symmetric matrices, they can provide the required fermion mass matrices and their mixings.

The second realization of $S U(3)$ is different, because it now requires a right handed neutrino to cancel anomaly. In the $S U(3)_{H}^{V}$ the fermions are vectorial. Both the left and right handed fermions transform as triplets under $S U(3)_{H}^{V}$ and all anti-fermions transform as anti-triplets. This implies that the standard model Higgs doublet that gives mass to the fermions after symmetry breaking could be a singlet $\phi^{c 1}[1,2,1,1]$ or an octet $\phi_{i j}^{c 2}[1,2,1,8]$ under $S U(3)_{H}^{V}$,

$$
\begin{aligned}
\mathcal{L}_{Y}= & g_{1}^{u} \delta_{i j} \bar{q}_{i L} u_{j R} \phi^{c 1^{\dagger}}+g_{1}^{d} \delta_{i j} \bar{q}_{i L} d_{j R} \phi^{c 1}+g_{1}^{e} \delta_{i j} \bar{\ell}_{i L} e_{j R} \phi^{c 1} \\
& +g_{8}^{u} \bar{q}_{i L} u_{j R} \phi_{i j}^{c 2}+g_{8}^{d} \bar{q}_{i L} d_{j R} \phi_{i j}^{c 2}+g_{8}^{e} \bar{\ell}_{i L} e_{j R} \phi_{i j}^{c 2}+h . c .
\end{aligned}
$$

The singlet Higgs $\phi^{c 1}$ gives equal masses to all generations with no mixing, but together with the octet Higgs they can produce all the required masses and mixing for the fermions. In the minimal model there are no new doublet Higgs scalar in addition to the $\phi^{c 1}$ and $\phi^{c 2}$. To break the $S U(3)_{H}^{V}$ horizontal symmetry some standard models singlet Higgs scalars are required which shall be discussed later. 
The main features of all the horizontal symmetries is that the Higgs doublets are required to be such that the generation structure comes only from the vevs of the doublet Higgs. The Yukawa couplings do not carry any generation index. We shall restrict ourselves to Higgs doublets which are required by the minimal models as discussed above.

We now turn to the question of neutrino masses in these horizontal symmetric models. The natural scenario of small neutrino masses is to have Majorana masses which are different realizations of a single effective operator [7],

$$
\mathcal{L}_{e f f}=y_{i j}^{e f f} \ell_{i L} \ell_{j L} \phi \phi .
$$

where $\phi$ is the usual Higgs doublet. The effective coupling $y_{i j}^{e f f}$ of this interaction includes the lepton number violating large scale $M$ in the denominator. If we write $y^{\text {eff }} \sim f / M$, then for $f \sim 10^{-3}$ we get $M \sim 10^{12} \mathrm{GeV}$ to explain the neutrino masses required by the atmospheric neutrino anomaly. For radiative models $f$ would have a smaller value and hence $M$ could be lower. The effective coupling $y^{e f f}$ will depend on the actual realization of this operator. The structure of the neutrino mass matrix

$$
\mathcal{M}_{\nu i j}=y_{i j}^{e f f}<\phi>^{2}
$$

depends completely on the effective Yukawa coupling.

The main point in our paper is that in theories with horizontal symmetries, the structure of the effective operators remain same, but the effective coupling is now completely independent of the generation structure. All the information about the generation structure are contained in the structure of the Higgs doublets. In other words, the structure of the neutrino mass matrix can be determined completely if we know how the Higgs doublets transform under the horizontal symmetric group. The only assumption we are making here is that for phenomenological reasons the horizontal symmetry is assumed to be broken at a scale much lower than the lepton number violating scale $M$. We assume that the mass of the gauge bosons of the horizontal symmetry 
is about $100 \mathrm{TeV}$, to have consistency with the lower bound on their mass coming from processes like $K_{L} \rightarrow \mu^{ \pm}+e^{\mp}$. If the horizontal symmetry is broken at a very high scale when the lepton number symmetry is also broken or higher, our result does not hold. Although we shall be discussing only few horizontal symmetry groups, but the above observation is true for any other horizontal symmetry groups including the abelian symmetries.

To understand this point, let us consider the example of see-saw mechanism [8]. In general the heavy neutrino mass matrix could have nontrivial structure in the generation space. But in case of horizontal symmetric models, at the lepton number violating scale the horizontal symmetries are still exact. So, the horizontal symmetries will prevent nontrivial structures of the heavy neutrino mass matrix. Only diagonal mass matrices are allowed for the right handed neutrinos.

In the minimal version of $S U(2)_{H}$, there is no right handed neutrino. One may add to the theory right handed neutrinos $N_{i R}$, which are singlets or even triplets under $S U(2)_{H}$. When the right handed neutrinos are three singlets, one may add a general Majorana mass term with nonvanishing off-diagonal terms. However, since $N_{i R}$ does not have any gauge interactions, without loss of generality we can diagonalise the mass matrix and work in a basis in which the heavy right handed neutrino mass matrix is diagonal.

In $S U(2)_{H}$ there are three standard model doublet Higgs scalars, a singlet $\phi^{a 1}$, a triplet $\phi_{i}^{a 2}$ and a 5 -plet $\phi_{i j}^{a 3}$. So for the singlet right handed neutrinos the Dirac neutrino masses could come only from the triplet Higgs scalar

$$
\mathcal{L}_{D}=f_{j}^{\nu} \bar{\ell}_{i L} N_{j R} \phi_{i}^{a 2^{\dagger}}
$$

If neutrino mass is generated through see-saw mechanism, the left handed neutrino mass matrix would come only from the triplet Higgs. We shall not restrict our discussions of neutrino masses to only one mechanism and hence we shall present a more general analysis.

The right handed neutrinos could also be triplets. We assumed that 
when the right handed neutrinos get mass at a scale $M$ breaking lepton number, the horizontal symmetry is exact. This implies that the right handed neutrino mass matrix comes from a singlet Higgs and hence the mass matrix is diagonal. Hence the low energy neutrino mass matrix again depends only on the standard model Higgs scalars appearing in the effective Majorana mass term and not on the effective Yukawa coupling constant $y^{\text {eff }}$. The Dirac neutrino mass term will now have contributions from all the Higgs scalars

$$
\mathcal{L}_{D}=f_{1}^{\nu} \delta_{i j} \bar{\ell}_{i L} N_{j R} \phi_{i}^{a \dagger}+f_{3}^{\nu} \epsilon_{i j k} \bar{\ell}_{i L} N_{j R} \phi_{k}^{a 2^{\dagger}}+f_{5}^{\nu} \bar{\ell}_{i L} N_{j R} \phi_{i j}^{a 3^{\dagger}}
$$

Thus vevs of all the Higgs scalars would contribute to the left handed Majorana neutrino mass matrix.

We can now come back to the effective operator contributing to the left handed neutrino Majorana mass matrix. These operators do not depend on the existence of the right handed neutrinos. Since the left handed neutrinos are triplets under $S U(2)_{H}$ the Majorana mass term $\ell_{i L} \ell_{j L}$ can be a singlet, triplet or a 5-plet. A singlet contribution can come from an operator $y^{e f f} \ell_{i L} \ell_{j L} \phi^{a 1} \phi^{a 1}$, but since this can give only a diagonal neutrino mass it is not sufficient. The triplet combination $y^{e f f} \epsilon_{i j k} \ell_{i L} \ell_{j L} \phi^{a 1} \phi_{k}^{a 12}$ vanishes identically since the neutrino mass matrix is symmetric. Thus only the 5plet combination is important. In the following we list all possible operators which can contribute to the 5-plet combination of the neutrino mass matrix

$$
\begin{aligned}
\mathcal{L}_{\text {eff }}^{1} & =y^{e f f} \ell_{i L} \ell_{j L} \phi_{i}^{a 2} \phi_{j}^{a 2} \\
\mathcal{L}_{\text {eff }}^{2} & =y^{e f f} \ell_{i L} \ell_{j L} \phi^{a 1} \phi_{i j}^{a 3} \\
\mathcal{L}_{\text {eff }}^{3} & =y^{\text {eff }} \ell_{i L} \ell_{j L} \phi_{i k}^{a 3} \phi_{j k}^{a 3} .
\end{aligned}
$$

An 5-plet of $S U(2)_{H}$ has isospin quantum number 2. Hence it has 5 projections, which are the 5-components. We can give vevs to any one of these components and hence there could be only 5 parameters that can determine 
the structure of the neutrino mass matrix. This is given by the matrix

$$
\left(\begin{array}{ccc}
a & b & c \\
b & \frac{c}{\sqrt{2}} & d \\
c & d & e
\end{array}\right) .
$$

The fact that the (22) component is same as the (13) or (31) component (modulo a factor of $\sqrt{2}$ ) restricts the possible textures of the neutrino mass matrix. Otherwise the mass matrix is very general and can explain the present data from atmospheric neutrino, solar neutrino and laboratory experiments for suitable value of the parameters coming from the vevs of the Higgs scalars [9]. The simplest choice would be when only the singlet give the charged lepton mass and hence diagonal. Otherwise we first have to diagonalise the charged lepton mass matrix to get the actual neutrino mass matrix.

For $S U(3)_{H}^{V L}$ one can only add singlet right handed neutrinos so as to give them Majorana mass with only $S U(3)_{H}^{V L}$ singlet Higgs scalars. Since these neutrinos do not have any gauge interaction, the mass matrix can be diagonalised without loss of generality. The Dirac mass of the neutrinos can then come from both the triplet $\tilde{\phi}_{[i j]}^{b 1}$ and anti-sextet $\tilde{\phi}_{\{i j\}}^{b 2}$ Higgs scalars

$$
\mathcal{L}_{D}=h_{3}^{\nu} \bar{\ell}_{i L} N_{j R} \tilde{\phi}_{[i j]}^{b 1^{\dagger}}+h_{6}^{u} \bar{\ell}_{i L} N_{j R} \tilde{\phi}_{\{i j\}}^{b 2^{\dagger}}
$$

The effective operator will not depend on the existence of the right handed neutrinos.

We now study the effective operators contributing to the Majorana neutrino masses for $S U(3)_{V L}$. Since the left handed neutrinos are triplets and the mass term is symmetric, only a sextet combination of the Higgs scalars would contribute to the effective operator. Possible operators are

$$
\begin{aligned}
\mathcal{L}_{\text {eff }}^{1} & =y^{e f f} \epsilon_{i k l} \epsilon_{j m n} \ell_{i L} \ell_{j L} \phi_{[k l]}^{b 1} \phi_{[m n]}^{b 1} \\
\mathcal{L}_{\text {eff }}^{1} & =y^{e f f} \epsilon_{i k m} \epsilon_{j l n} \ell_{i L} \ell_{j L} \phi_{\{k l\}}^{b 2} \phi_{\{m n\}}^{b 2}
\end{aligned}
$$


Since the effective mass term is a sextet, it is given by 6 parameters in the matrix

$$
\left(\begin{array}{lll}
a & b & c \\
b & d & e \\
c & e & f
\end{array}\right) .
$$

It is thus clear that this mass matrix is the most general symmetric mass matrix and hence can explain any experiment with proper choice of these six parameters, which are given by the vevs of the Higgs doublets.

The $S U(3)_{H}^{V}$ case is most interesting. As in the $S U(3)_{H}^{V L}$ case, the right handed neutrinos can only be singlets, otherwise they cannot get Majorana mass before $S U(3)_{H}^{V}$ is broken. The left handed leptons are again triplet, so the Dirac mass term would require a triplet Higgs scalar, which is not there in the model. Hence the right handed neutrinos decouple from the left handed neutrino mass matrix. We shall come back to this point later.

Since the left handed neutrinos are triplets, the effective mass term is a sextet as in the previous case. But now the Higgs scalars in the minimal model are singlet and octet. Hence there are no dimension five operators, which can allow neutrino masses. If we now include a Higgs, which is standard model singlet and triplet under $S U(3)_{H}^{V} \eta_{\{i j\}}[1,1,0, \overline{6}]$, which can break the horizontal symmetry group, then we can have the effective operators

$$
\begin{aligned}
\mathcal{L}_{\text {eff }}^{1} & =y^{e f f} \ell_{i L} \ell_{j L} \phi^{c 1} \phi^{c 1} \eta_{\{i j\}} \\
\mathcal{L}_{\text {eff }}^{2} & =y^{e f f} \ell_{i L} \ell_{j L} \phi_{k l}^{c 2} \phi_{i l}^{c 2} \eta_{\{k j\}} \\
\mathcal{L}_{\text {eff }}^{3} & =y^{e f f} \ell_{i L} \ell_{j L} \phi^{c 1} \phi_{i k}^{c 2} \eta_{\{k j\}} .
\end{aligned}
$$

Now the operators are of dimension 6 and hence suppressed by one extra power of the lepton number violating scale $M$. In case of dimension 5 operators the lepton number violating scale was $M \sim 10^{12} \mathrm{GeV}$. But now the required lepton number violating scale would be about $M \sim 10^{8} \mathrm{GeV}$. Since we cannot construct a dimension 5 operator in this case, it is obvious the mechanism for neutrino mass has to be somewhat different from the canon- 
ical case. This is clear from the fact that there is no Dirac mass term now (which will immediately forbid the see-saw mechanism). For example, one can now generate a neutrino mass in the triplet Higgs mechanism [10], where the triplet Higgs is a sextet under $S U(3)_{H}^{V}$. This result will change if one considers nonminimal model by including a sextet and triplet standard model doublet Higgs scalars.

The lepton number violating scale could be further lowered, if the horizontal symmetry is broken by a triplet Higgs $\omega_{i}[1,1,0, \overline{3}]$ instead of a sextet Higgs. In this case the effective operators are

$$
\begin{aligned}
\mathcal{L}_{\text {eff }}^{1} & =y^{\text {eff }} \ell_{i L} \ell_{j L} \phi^{c 1} \phi^{c 1} \omega_{i} \omega_{j} \\
\mathcal{L}_{\text {eff }}^{2} & =y^{e f f} \ell_{i L} \ell_{j L} \phi_{k l}^{c 2} \phi_{i l}^{c 2} \omega_{k} \omega_{j} \\
\mathcal{L}_{\text {eff }}^{3} & =y^{\text {eff }} \ell_{i L} \ell_{j L} \phi^{c 1} \phi_{i k}^{c 2} \omega_{k} \omega_{j} .
\end{aligned}
$$

These operators have dimension 7 and hence they are suppressed by third power of lepton number violating scale. This implies that the lepton number violating scale $M$ could be as low as $M \sim 1000 \mathrm{TeV}$. In this case the left handed neutrino mass would be determined by the sextet structure of the effective Higgs and hence the mass matrix would appear to be similar to $S U(3)_{H}^{V L}$ model.

In summary, we pointed out that in models with gauged horizontal symmetries it is possible to get the structure of the neutrino mass matrix without knowing the mechanism which generates the neutrino mass. This is because the effective operator for the neutrino masses contains all the generation indices in the Higgs vevs and the effective Yukawa coupling is independent of the generation structure. We studied the neutrino mass matrices in the $S U(2)_{H}$ and , $S U(3)_{H}^{V}$ and $S U(3)_{H}^{V L}$ horizontal symmetric models.

\section{References}


[1] S. Weinberg, in a Festschrift for I. I. Rabi, ed. L. Motz (N.Y. Academy of Sciences, N.Y. 1977); A. deRejula, H. Georgi, and S. L. Glashow, Ann. Phys. (N.Y.) 109, 258 (1977); F. Wilczek and A. Zee, Phys. Lett. B79, 418 (1977); H. Georgi and D. V. Nanopoulos, Nucl. Phys. B155, $52(1979)$.

[2] J.C. Pati, A. Salam, J. Strathdee, Phys. Lett. B 58, 265 (1975); B 108, 121 (1982); H. Terazawa, Prog. Theor. Phys. 58, 1276 (1977); F. Wilczek and A. Zee, Phys. Rev. Lett. 42, 421 (1979); T. Maehara and T. Yanagida, Prog. Theor. Phys. 61, 1434 (1979); D.R.T. Jones, G.L. Kane and J.P. Leveille, Nucl. Phys. B 198, 45 (1982); A.K. Ray and U. Sarkar, Phys. Rev. D 29, 166 (1984)(RC); K. Bandyopadhyay, A.K. Ray and U. Sarkar, Phys. Lett. B 151, 132 (1985); Phys. Rev. D 33, 3293 (1986).

[3] Super-Kamiokande Collaboration : Y. Fukuda et al, Phys. Rev. Lett. 81, 1562 (1998); Phys. Lett. B433, 9 (1998); Phys. Rev. Lett. 85, 3999 (2000).

[4] Super-Kamiokande Collaboration: Y. Fukuda et al, Phys. Rev. Lett. 81, 1158 (1998); Phys. Rev. Lett. 86, 5656 (2001); SNO Collaboration: Q.R. Ahmad, et. al., Phys. Rev. Lett. 87, 071301 (2001).

[5] K2K Collaboration: S.H. Ahn, et. al., Phys. Lett. B 511, 178 (2001); K. Nishikawa, im Proc. of the Int. Europhysics Conf. on High Energy Physics (Budapest, Hungary, July 2001); C.K. Jung, in Proc. of the XX Int. Symp. on lepton and Photon Interactions at High Energies (Rome, Italy, July 2001).

[6] LSND Collaboration: C. Athanassopoulos, et. al., Phys. Rev. Lett. 77, 3082 (1996); Phys. Rev. Lett. 81, 1774 (1998). 
[7] S. Weinberg, Phys. Rev. Lett. 43, 1566 (1979); F. Wilczek and A. Zee, Phys. Rev. Lett. 43, 1571 (1979); E. Ma, Phys. Rev. Lett. 81, 1171 (1998);

[8] M. Gell-Mann, P. Ramond and R. Slansky, in Supergravity, eds. P. van Nieuwenhuizen and D. Freedman, (North-Holland, 1979) p.315; T. Yanagida, in Proc. Workshop on Unified Theories and Baryon Number in the Universe, eds. A. Sawada and A. Sugamoto (KEK, 1979), p.95; R.N. Mohapatra and G. Senjanovic, Phys. Rev. Lett. 44, 912 (1980).

[9] V. Barger, et. al., Phys. Rev. D 58, 093016 (1998); Phys. Lett. B 437, 107 (1998); Phys. Rev. Lett. 82, 2640 (1999); Phys. Lett. B 462, 109 (1999); G. Altarelli and F. Feruglio, Phys. Lett. B 439, 112 (1998); B 451, 388 (1999); Phys. Rep. 320, 295 (1999); R. Barbieri, et. al., Phys. Lett. B 445, 407 (1999); JHEP 9812:017 (1998); D.P. Roy et. al., Phys. Lett. B 445, 185 (1998); B 444, 391 (1998); Phys. Rev. D 59, 097702 (1999); G.L. Fogli, et. al., Phys. Rev. D 59, 033001 (1999); Nucl. Phys. Proc. Suppl. 70, 261 (1999); Nucl. Phys. Proc. Suppl. 85, 159 (2000); D.V. Ahluwalia, et. al., Mod. Phys. Lett. A 13, 2249 (1998); Phys. Lett. B 460, 431 (1999); U. Sarkar, Phys. Rev. D 59, 037302 (1999); Phys. Rev. D 59, 031301 (1999); R.N. Mohapatra and S. Nussinov, Phys. Lett. B 346, 75 (1995); Phys. Lett. B 441, 299 (1998); Phys. Rev. D 60, 013002 (1999).

[10] E. Ma et. al., Phys. Rev. Lett. 80, 5716 (1998); Nucl. Phys. B 602, 23 (2001). 